Article

\title{
Set Evincing the Ranks with Respect to an Embedded Variety (Symmetric Tensor Rank and Tensor Rank
}

\author{
Edoardo Ballico \\ Department of Mathematics, University of Trento, 38123 Povo, Italy; edoardo.ballico@unitn.it
}

Received: 11 July 2018; Accepted: 8 August 2018; Published: 14 August 2018

\begin{abstract}
Let $X \subset \mathbb{P}^{r}$ be an integral and non-degenerate variety. We study when a finite set $S \subset X$ evinces the $X$-rank of the general point of the linear span of $S$. We give a criterion when $X$ is the order $d$ Veronese embedding $X_{n, d}$ of $\mathbb{P}^{n}$ and $|S| \leq\left(\begin{array}{c}n+\lfloor d / 2\rfloor \\ n\end{array}\right)$. For the tensor rank, we describe the cases with $|S| \leq 3$. For $X_{n, d}$, we raise some questions of the maximum rank for $d \gg 0$ (for a fixed $n$ ) and for $n \gg 0$ (for a fixed $d$ ).
\end{abstract}

Keywords: $X$-rank; symmetric tensor rank; tensor rank; veronese variety; segre variety

\section{Introduction}

Let $X \subset \mathbb{P}^{r}$ be an integral and non-degenerate variety. For any $q \in \mathbb{P}^{r}$, the $X$-rank $r_{X}(q)$ of $q$ is the minimal cardinality of a finite set $S \subset X$ such that $q \in\langle S\rangle$, where \langle\rangle denotes the linear span. The definition of $X$-ranks captures the notion of tensor rank (take as $X$ the Segre embedding of a multiprojective space) of rank decomposition of a homogeneous polynomial (take as $X$ a Veronese embedding of a projective space) of partially symmetric tensor rank (take a complete linear system of a multiprojective space) and small variations of it may be adapted to cover other applications. See [1] for many applications and [2] for many algebraic insights. For the pioneering works on the applied side, see, for instance, [3-7]. The paper [7] proved that $X$-rank is not continuous and showed why this has practical importance. The dimensions of the secant varieties (i.e., the closure of the set of all $q \in \mathbb{P}^{r}$ with a prescribed rank) has a huge theoretical and practical importance. The Alexander-Hirschowitz theorem computes in all cases the dimensions of the secant varieties of the Veronese embeddings of a projective space ([8-14]). For the dimensions of secant varieties, see [15-17] for tensors and [18-27] for partially symmetric tensors (i.e., Segre-Veronese embeddings of multiprojective spaces). For the important problem of the uniqueness of the set evincing a rank (in particular for the important case of tensors) after the classical [28], see [29-38]. See [39-47] for other theoretical works.

Let $S \subset X$ be a finite set and $q \in \mathbb{P}^{r}$. We say that $S$ evinces the X-rank of $q$ if $q \in\langle S\rangle$ and $|S|=r_{X}(q)$. We say that $S$ evinces an X-rank if there is $q \in \mathbb{P}^{r}$ such that $S$ evinces the $X$-rank of $q$. Obviously, $S$ may evince an $X$-rank only if it is linearly independent, but this condition is not a sufficient one, except in very trivial cases, like when $r_{X}(q) \leq 2$ for all $q \in \mathbb{P}^{r}$. Call $r_{X, \max }$ the maximum of all integers $r_{X}(q)$. An obvious necessary condition is that $|S| \leq r_{X \text {,max }}$ and this is in very special cases a sufficient condition (see Propositions 1 for the rational normal curve). If $S$ evinces the $X$-rank of $q \in \mathbb{P}^{r}$, then $q \in\langle S\rangle$ and $q \notin\left\langle S^{\prime}\right\rangle$ for any $S^{\prime} \subsetneq S$. For any finite set $S \subset \mathbb{P}^{r}$, set $\langle S\rangle^{\prime}:=\langle S\rangle \backslash\left(\cup_{S^{\prime} \subsetneq S}\left\langle S^{\prime}\right\rangle\right)$. Note that $\langle S\rangle^{\prime}=\varnothing$ if and only if either $S=\varnothing$ or $S$ is linearly dependent (when $|S|=1,\langle S\rangle^{\prime}=S$ and $S$ evinces itself). In some cases, it is possible to show that some finite $S \subset X$ evinces the $X$-rank of all points of $\langle S\rangle^{\prime}$. We say that $S$ evinces generically the 
$X$-ranks if there is a non-empty Zariski open subset $U$ of $\langle S\rangle$ such that $S$ evinces the $X$-ranks of all $q \in U$. We say that $S$ totally evinces the $X$-ranks if $S$ evinces the $X$-ranks of all $q \in\langle S\rangle^{\prime}$. We first need an elementary and well-known bound to compare it with our results.

Let $\rho(X)$ be the maximal integer such that each subset of $X$ with cardinality $\rho(X)$ is linearly independent. See ([43] Lemma 2.6, Theorem 1.18) and ([42] Proposition 2.5) for some uses of the integer $\rho(X)$. Obviously, $\rho(X) \leq r+1$ and it is easy to check and well known that equality holds if and only if $X$ is a Veronese embedding of $\mathbb{P}^{1}$ (Remark 1 ). If $|S| \leq\lfloor(\rho(X)+1) / 2\rfloor$, then $S$ totally evinces the $X$-ranks (as in [43] Theorem 1.18) while, for each integer $t>\lfloor(\rho(X)+1) / 2\rfloor$ with $t \leq r+1$, there is a linearly independent subset of $X$ with cardinality $t$ and not totally evincing the $X$-ranks ( Lemma 3). Thus, to say something more, we need to make some assumptions on $S$ and these assumptions must be related to the geometry of $X$ or the reasons for the interest of the $X$-ranks. We do this in Section 3 for the Veronese embeddings and in Section 4 for the tensor rank. For tensors, we only have results for $|S| \leq 3$ (Propositions 3 and 4 ).

For all positive integers $n, d$ let $v_{d, n}: \mathbb{P}^{n} \rightarrow \mathbb{P}^{r}, r=\left(\begin{array}{c}n+d \\ n\end{array}\right)-1$, denote the Veronese embedding of $\mathbb{P}^{n}$, i.e., the embedding of $\mathbb{P}^{n}$ induced by the complete linear system $\left|\mathcal{O}_{\mathbb{P}^{n}}(d)\right|$. Set $X_{n, d}:=$ $v_{d, n}\left(\mathbb{P}^{n}\right)$. At least over an algebraically closed base field of characteristic 0 (i.e., in the set-up of this paper), for any $q \in \mathbb{P}^{r}$, the integer $r_{X_{n, d}}(q)$ is the minimal number of $d$-powers of linear forms in $n+1$ variables whose sum is the homogeneous polynomial associated to $q$.

We prove the following result, whose proof is elementary (see Section 3 for the proof). In its statement, the assumption " $h^{1}\left(\mathcal{I}_{A}(\lfloor d / 2\rfloor)\right)=0$ " just means that the vector space of all degree $\lfloor d / 2\rfloor$ homogeneous polynomials in $n+1$ variables vanishing on $A$ has dimension $\left(\frac{n+\lfloor d / 2\rfloor}{n}\right)-|A|$, i.e., $A$ imposes $|A|$ independent conditions to the homogeneous polynomials of degree $\lfloor d / 2\rfloor$ in $n+1$ variables.

Theorem 1. Fix integers $n \geq 2, d>k>2$ and a finite set $A \subset \mathbb{P}^{n}$ such that $h^{1}\left(\mathcal{I}_{A}(\lfloor d / 2\rfloor)\right)=0$. Set $S:=v_{d, n}(A)$. Then, $S$ totally evinces the ranks for $X_{n, d}$.

A general $A \subset \mathbb{P}^{n}$ satisfies the assumption of Theorem 1 if and only if $|A| \leq\left(\begin{array}{c}n+\lfloor d / 2\rfloor \\ n\end{array}\right)$. For much smaller $|A|$, one can check the condition $h^{1}\left(\mathcal{I}_{A}(\lfloor d / 2\rfloor)\right)=0$ if $A$ satisfies some geometric conditions (e.g., if $A$ is in linearly general position, it is sufficient to assume $|A| \leq n\lfloor d / 2\rfloor+1$ ).

We conclude the paper with some questions related to the maximum of the $X$-ranks when $X$ is a Veronese embedding of $\mathbb{P}^{n}$.

\section{Preliminary Lemmas}

Remark 1. Let $X \subset \mathbb{P}^{r}$ be an integral and non-degenerate variety. Since any $r+2$ points of $\mathbb{P}^{r}$ are linearly dependent, we have $\rho(X) \leq r+1$. If $X$ is a rational normal curve, then $\rho(X)=r+1$ because any $r+1$ points of $X$ spans $\mathbb{P}^{r}$. Now, we check that, if $\rho(X)=r+1$, then $X$ is a rational normal curve. This is well known, but usually stated in the set-up of Veronese embeddings or the X-ranks of curves. Set $n:=\operatorname{dim} X$ and $d:=\operatorname{deg}(X)$. Assume $\rho(X)=r+1$. Let $H \subset \mathbb{P}^{r}$ be a general hyperplane. If $n>1$, then $X \cap H$ has dimension $n-1>0$ and in particular it has infinitely many points. Any $r+1$ points of $X \cap H$ are linearly dependent. Now, assume $n=1$. Since $X$ is non-degenerate, we have $d \geq n$. By Bertini's theorem, $X \cap H$ contains $d$ points of $X$. Since $\rho(X)=r+1, \operatorname{dim} H=r-1$ and $H \cap X \subset H$, we have $d \leq r$. Hence, $d=r$, i.e., $X$ is a rational normal curve.

The following example shows, that in many cases, there are are sets evincing $X$-ranks, but not totally evincing $X$-ranks or even generically evincing $X$-ranks.

Example 1. Let $X \subset \mathbb{P}^{r}, r \geq 3$, be a rational normal curve. Take $q \in \mathbb{P}^{r}$ with $r_{X}(q)=r$, i.e., take $q \in \tau(X) \backslash X$, where $\tau(X)$ is the tangential variety of $X([48])$. Take $S \subset X$ evincing the $X$-rank of $q$. Thus, $|S|=r$ and $S$ spans a hyperplane $\langle S\rangle$. Since $\operatorname{dim} \tau(X)=2$ and $\tau(X)$ spans $\mathbb{P}^{r},\langle S\rangle \cap \tau(X)$ is a 
proper closed algebraic subset of $\langle S\rangle$. Thus, for a general $p \in\langle S\rangle$, we have $r_{X}(p)<|S|$ and hence $S$ does not generically evinces $X$-ranks.

Lemma 1. If $S \subset X$ is a finite set evincing the rank of some $q \in \mathbb{P}^{r}$, then each $S^{\prime} \subset S, S^{\prime} \neq \varnothing$, evinces the $X$-rank of some $q^{\prime} \in \mathbb{P}^{r}$.

Proof. We may assume $S^{\prime} \neq S$. Write $S^{\prime \prime}:=S \backslash S^{\prime}$. Since $S$ evinces the rank of $q, S$ is linearly independent, but $S \cup\{q\}$ is not linearly independent. Since $S^{\prime} \neq \varnothing$ and $S^{\prime \prime} \neq \varnothing$, there are unique $q^{\prime} \in\left\langle S^{\prime}\right\rangle$ and $q^{\prime \prime} \in\left\langle S^{\prime \prime}\right\rangle$ such that $q \in\left\langle\left\{q^{\prime}, q^{\prime \prime}\right\}\right\rangle$. Since $S$ evinces the rank of $q, S^{\prime}$ evinces the rank of $q^{\prime}$.

Lemma 2. Every non-empty subset of a set evincing generically (resp. totally) X-ranks evinces generically (resp. totally) the X-ranks.

Proof. Assume that $S$ evinces generically the $X$-ranks and call $U$ a non-empty open subset of $\langle S\rangle^{\prime}$ such that $r_{X}(q)=|S|$ for all $q \in U$; if $S$ evinces totally the $X$-ranks, take $U:=\langle S\rangle^{\prime}$. Fix $S^{\prime} \subsetneq S$, $S^{\prime} \neq 0$ and set $S^{\prime \prime}:=S \backslash S^{\prime}$. Let $E$ be the set of all $q \in\langle S\rangle^{\prime}$ such that $\left\langle\{q\} \cup S^{\prime \prime}\right\rangle \cap U \neq \varnothing$. If $q \in E$, then $r_{X}(q)=\left|S^{\prime}\right|$ because $r_{X}\left(q^{\prime}\right)=|S|$ for each $q^{\prime} \in\left\langle\{q\} \cup S^{\prime \prime}\right\rangle \cap U$. Since $S^{\prime} \cap S^{\prime \prime}=\varnothing$ and $S^{\prime} \cup S^{\prime \prime}=S$ is linearly independent, $E$ is a non-empty open subset of $\langle S\rangle^{\prime}$ (a general element of $\langle S\rangle$ is contained in the linear span of a general element of $\left\langle S^{\prime}\right\rangle$ and a general element of $\left\langle S^{\prime}\right\rangle$ ). Now, assume $U=\langle S\rangle^{\prime}$. Every element of $\langle S\rangle^{\prime}$ is in the linear span of an element of $\left\langle S^{\prime}\right\rangle^{\prime}$ and an element of $\left\langle S^{\prime \prime}\right\rangle^{\prime}$.

Lemma 3. Take a finite set $S \subset X, S \neq \varnothing$.

(a) If $|S| \leq\lfloor(\rho(X)+1) / 2\rfloor$, then $S$ totally evinces the X-ranks.

(b) For each integer $t>\lfloor(\rho(X)+1) / 2\rfloor$, there is $A \subset X$ such that $|A|=t$ and $A$ does not totally evince the X-ranks.

Proof. Take $q \in\langle S\rangle^{\prime}$ and assume $r_{X}(q)<|S|$. Take $B \subset X$ evincing the $X$-rank of $q$. Since $|B|<|S|$, we have $B \neq S$. Since $q \in\langle S\rangle \cap\langle B\rangle$, but no proper subset of either $B$ or $S$ spans $q, S \cup B$ is linearly dependent. Since $|B| \leq|S|-1$, we have $|B \cup S| \leq \rho(X)$, contradicting the definition of $\rho(X)$.

Now, we prove part (b). By Lemma 1, it is sufficient to do the case $t=\lfloor(\rho(X)+1) / 2\rfloor+1$. By the definition of the integer $\rho(X)$, there is a subset $D \subset X$ with $|D|=\rho(X)+1$ and $D$ linearly dependent. Write $D=A \sqcup E$ with $|A|=\lfloor(\rho(X)+1) / 2\rfloor+1$ and $|E|=\rho(X)+1-|A|$. Note that $|A|>|E|$. Since $|A| \leq \rho(X)$ (remember that $\rho(X) \geq 2$ ), both $A$ and $E$ are linearly independent. Since $A \cup E$ is linearly dependent, there is $q \in\langle A\rangle \cap\langle E\rangle$. Since $|D|=\rho(X)+1$, every proper subset of $D$ is linearly independent. Hence, $\left\langle A^{\prime}\right\rangle \cap\langle E\rangle=\varnothing$ for all $A^{\prime} \subsetneq A$. Thus, $q \in\langle A\rangle^{\prime}$. Since $|E|<|A|, A$ does not evince the $X$-rank of $q$.

Remark 2. Take $X \subset \mathbb{P}^{r}$ such that $r_{X}(q) \leq 2$ for all $q \in \mathbb{P}^{r}$ (e.g., by [49], we may take most space curves). Any set $S \subset X$ with $|S|=2$ evinces its $X$-ranks if and only if $X$ contains no line.

\section{The Veronese Embeddings of Projective Spaces}

Let $v_{d, n}: \mathbb{P}^{n} \rightarrow \mathbb{P}^{r}, r:=-1+\left(\begin{array}{c}n+d \\ n\end{array}\right)$, denote the Veronese embedding of $\mathbb{P}^{n}$. Set $X_{n, d}:=$ $v_{d, n}\left(\mathbb{P}^{n}\right)$.

Proposition 1. Let $X \subset \mathbb{P}^{d}, d \geq 2$, be the rational normal curve.

(a) A non-empty finite set $S \subset X$ evinces some rank of $\mathbb{P}^{d}$ if and only if $|S| \leq d$.

(b) A non-empty finite set $A \subset X$ totally evinces the X-ranks if and only if $|A| \leq\lfloor(d+2) / 2\rfloor$. 
Proof. By a theorem of Sylvester's ([48]), every $q \in \mathbb{P}^{d}$ has X-rank at most $d$. Thus, the condition $|S| \leq d$ is a necessary condition for evincing some rank. By Lemma 1 to prove part (a), it is sufficient to prove it when $|S|=d$. Take any connected zero-dimensional scheme $Z \subset X$ with $\operatorname{deg}(Z)=2$ and $S \cap Z=\varnothing$. Thus, $\operatorname{deg}(Z \cup S)=d+2$. Since $X \cong \mathbb{P}^{1}, \operatorname{deg}\left(\mathcal{O}_{X}(1)\right)=d$ and $X$ is projectively normal, we have $h^{1}\left(\mathcal{I}_{S \cup Z}(1)\right)=1$ and $h^{1}\left(\mathcal{I}_{W}(1)\right)=0$ for each $W^{\prime} \subsetneq S \cup Z$. This is equivalent to say that the line $\langle Z\rangle$ meets $\langle S\rangle$ at a unique point, $q$ and $q \neq Z_{\text {red }}$. By Sylvester's theorem, $r_{X}(q)=d$ ([48]). Since $q \in\langle S\rangle$ and $|S|=d$, $S$ evinces the $X$-rank of $q$.

If $A \neq \varnothing$ and $|A| \leq\lfloor(d+2) / 2\rfloor$, then $A$ totally evinces the $X$-ranks by part (a) of Lemma 3 and the fact that $\rho(X)=d+1$. Now, assume $d \geq|A|>\lfloor(d+2) / 2\rfloor$. Fix a set $E \subset X \backslash A$ with $|E|=d+2-|A|$. Adapt the proof of part (b) of Lemma 3 .

Proposition 2. Fix a set $S \subset X_{n, d}, n \geq 2$, with $|S|=d+1$. The following conditions are equivalent:

1. there is a line $L \subset \mathbb{P}^{n}$ such that $|S \cap L|>\lfloor(d+2) / 2\rfloor$;

2. S evinces no $X_{n, d^{-}}$rank;

3. there is $q \in\langle S\rangle^{\prime}$ such that $S$ does not evince the $X_{n, d}$-rank of $q$.

Proof. Obviously, (2) implies (3). If $X^{\prime} \subset X$ is a subvariety and $q \in\left\langle X^{\prime}\right\rangle$, we have $r_{X^{\prime}}(q) \geq r_{X}(q)$. Thus, Sylvester's theorem ([48]) and Lemma 2 show that (1) implies (2).

Now, assume the existence of $q \in\langle S\rangle^{\prime}$ such that $S$ does not evince the $X$-rank of $q$, i.e., $r_{X}(q) \leq d$. Take $A \subset \mathbb{P}^{n}$ such that $v(A)=S$ and take $B \subset \mathbb{P}^{n}$ such that $v_{d}(B)$ evinces the $X$-rank of $q$. Since $q \in\langle S\rangle^{\prime}$, (Ref. [50] Lemma 1) gives $h^{1}\left(\mathbb{P}^{n}, \mathcal{I}_{A \cup B}(d)\right)>0$. Since $|A \cup B| \leq 2 d+1$, (Ref. [51] Lemma 34) gives the existence of a line $L \subset \mathbb{P}^{n}$ such that $|L \cap(A \cup B)| \geq d+2$. Let $H \subset \mathbb{P}^{n}$ be a general hyperplane containing $L$. Since $H$ is general and $A \cup B$ is a finite set, we have $H \cap(A \cup B)=L \cap(A \cup B)$. Since $|L \cap(A \cup B)| \geq d+2$, we have $|A \cup B \backslash H \cap(A \cup B)| \leq d-1$ and hence $\left.h^{1}\left(\mathbb{P}^{n}, \mathcal{I}_{A \cup B \backslash H \cap(A \cup B}\right)(d-1)\right)=0$. By ([52] Lemma 5.2), we have $A \backslash A \cap H=B \backslash B \cap H$.

See $[53,54]$ for some results on the geometry of sets $S \subset X_{n, d}$ with controlled Hilbert function and that may be useful to extend Proposition 2.

Proof of Theorem 1: Set $k:=\lfloor d / 2\rfloor$. Note that $h^{1}\left(\mathcal{I}_{A}(x)\right)=0$ for all $x \geq k$ and in particular $h^{1}\left(\mathcal{I}_{A}(d-k)\right)=0$. Fix $q \in\left\langle v_{d, n}(A)\right\rangle^{\prime}$ and assume $r_{X_{n, d}}(q)<|A|$. Fix $B \subset \mathbb{P}^{n}$ such that $v_{d, n}(B)$ evinces the $X_{n, d}$-rank of $q$. Since $h^{1}\left(\mathcal{I}_{A}(k)\right)=0$ and $|A|>|B|$, we have $h^{0}\left(\mathcal{I}_{B}(k)\right)>h^{0}\left(\mathcal{I}_{A}(k)\right)$. Thus, there is $M \in\left|\mathcal{O}_{\mathbb{P}}(k)\right|$ containing $B$, but with $A \nsubseteq M$, i.e., $A \backslash A \cap M \neq \varnothing$, while $B \backslash B \cap M=$ $\varnothing$. Since $h^{1}\left(\mathcal{I}_{A}(d-k)\right)=0$, we have $h^{1}\left(\mathcal{I}_{A \backslash A \cap M}(d-k)\right)=0$. Since $h^{1}\left(\mathcal{I}_{A}(d)\right)=0, v_{d, n}(A)$ is linearly independent. Since $v_{d, n}(B)$ evinces a rank, it is linearly independent. Grassmann's formula gives $\operatorname{dim}\left\langle v_{d, k}(A)\right\rangle \cap\left\langle v_{d, b}(B)\right\rangle=|A \cap B|+h^{1}\left(\mathcal{I}_{A \cup B}(d)\right)-1$. We have $A \cup B=((A \cup B) \cap M) \cup$ $(A \backslash A \cap M)$. Since $A \backslash A \cap B$ is a finite set, we have $h^{2}\left(\mathcal{I}_{A \backslash A \cap B}(d-k)\right)=h^{2}\left(\mathcal{O}_{\mathbb{P}^{n}}(d-k)\right)=0$. Since $h^{1}\left(\mathcal{I}_{A \backslash A \cap M}(d-k)\right)=0$, the residual exact sequence (also known as the Castelnuovo's sequence)

$$
0 \rightarrow \mathcal{I}_{A \backslash A \cap B}(d-k) \rightarrow \mathcal{I}_{A \cup B}(d) \rightarrow \mathcal{I}_{M \cap(A \cup B), M}(d) \rightarrow 0
$$

gives $h^{1}\left(\mathcal{I}_{A \cup B}(d)\right)=h^{1}\left(M, \mathcal{I}_{M \cap(A \cup B)}(d)\right)$. Since $M$ is projectively normal, $h^{1}\left(M, \mathcal{I}_{M \cap(A \cup B)}(d)\right)=$ $h^{1}\left(\mathcal{I}_{A \cup B}(d)\right)$. Thus, the Grassmann's formula gives $\operatorname{dim}\left\langle v_{d, n}(A \cap M)\right\rangle \cap\left\langle v_{d, n}(B \cap M)\right\rangle=\mid A \cap B \cap$ $M \mid+h^{1}\left(\mathcal{I}_{A \cup B}(d)\right)-1$. Since $B \subset M$, we get $\left\langle v_{d, n}(A \cap M)\right\rangle \cap\left\langle v_{d, n}(B \cap M)\right\rangle=\left\langle v_{d, k}(A)\right\rangle \cap\left\langle v_{d, b}(B)\right\rangle$. Since $A \cap M \supsetneq A$, we get $q \notin\left\langle v_{d, n}(A)\right\rangle^{\prime}$, a contradiction.

\section{Tensors, i.e., the Segre Varieties}

Fix an integer $k \geq 2$ and positive integers $n_{1}, \ldots, n_{k}$. Set $Y:=\prod_{i=1}^{k} \mathbb{P}^{n_{i}}$ (the Segre variety) and $N:=-1+\prod_{i=1}^{k}\left(n_{i}+1\right)$. Let $v: Y \rightarrow \mathbb{P}^{N}$ denote the Segre embedding. Let $\pi_{i}: Y \rightarrow \mathbb{P}^{n_{i}}$ denote the projection on the $i$-th factor. For any $i \in\{1, \ldots, k\}$, set $Y[i]:=\prod_{h \neq i} \mathbb{P}^{n_{h}}$ and call $\eta_{i}: Y \rightarrow Y[i]$ the 
projection which forgets the $i$-th component. Let $v[i]: Y[i] \rightarrow \mathbb{P}^{N_{i}}, N_{i}:=-1+\prod_{h \neq i}\left(n_{h}+1\right)$ denote the Segre embedding of $Y[i]$. A key difficulty is that $\rho(v(Y))=2$ because $v(Y)$ contains lines.

Lemma 4. Let $S \subset Y$ be any finite set such that there is $i \in\{1, \ldots k\}$ with $\eta_{i \mid S}$ not injective. Then, $v(S)$ evinces no rank.

Proof. By Lemma 1, we reduce to the case $|S|=2$, say $S=\{a, b\}$ with $a=\left(a_{1}, \ldots, a_{k}\right)$, $b=\left(b_{1}, \ldots, b_{k}\right)$ with $a_{i}=b_{i}$ if and only if $i>1$. Since all lines of $Y$ are contained in one of the factors of $Y$ and all lines of $v(Y)$ are images of lines of $Y$, we get $S \subset v(Y)$. Thus, each element of $\langle v(S)\rangle$ is contained in $v(Y)$ and hence it has rank 1. Since $|S|>1, v(S)$ evinces no rank.

Lemma 5. Let $S \subset Y$ such that there are $S^{\prime} \subseteq S$ and $i \in\{1, \ldots, k\}$ with $\left|S^{\prime}\right|=3, v_{i}\left(\eta_{i}\left(S^{\prime}\right)\right)$ linearly dependent and $\pi_{i}\left(S^{\prime}\right) \subset \mathbb{P}^{n_{i}}$ linearly dependent. Then, $v(S)$ evinces no rank.

Proof. Let $Q \subset \mathbb{P}^{3}$ be a smooth quadric surface. $Q$ is projectively equivalent to the Segre embedding of $\mathbb{P}^{1} \times \mathbb{P}^{1}$ and each point of $\mathbb{P}^{3}$ has at most $Q$-rank 2 by [47] (Proposition 5.1). By Lemma 1, we may assume $S^{\prime}=S$. By Lemma 4, we may assume that $\eta_{i \mid S}$ is injective. Thus, $\left|\eta_{i \mid S}\right|=3$. Since $v_{i}\left(\eta_{i}(S)\right)$ is not linearly independent and it has cardinality 3 , it is contained in a line of $v_{i}(Y[i])$. Thus, $\eta_{i}(S)$ is contained in a line of one of the factors of $Y[i]$. By assumption, $\pi_{i}(S)$ is contained in a line of $\mathbb{P}^{n_{i}}$. Thus, $S$ is contained in a subscheme of $Y$ isomorphic to $\mathbb{P}^{1} \times \mathbb{P}^{1}$. Since each point of $\mathbb{P}^{3}$ has $Q$-rank $\leq 2$ and $|S|=3, v(S)$ evinces no rank.

Remark 3. Fix a finite set $A \subset Y$ such that $S:=v(A)$ is linearly independent. $S$ evinces no tensor rank if there is a multiprojective subspace $Y^{\prime} \subset Y$ such that $A \subset Y^{\prime}$ and $|S|$ is larger than the maximum tensor $\operatorname{rank}$ of $v\left(Y^{\prime}\right)$.

Note that Lemmas 4 and 5 may be restated as a way to check for very low $|S|$ if there is some $Y^{\prime}$ as in Lemma 3 exists.

Proposition 3. Take $S \subset v(Y)$ with $|S|=2$. Let $Y^{\prime}$ be the minimal multiprojective subspace of $Y$ containing $S$. The following conditions are equivalent:

1. S evinces no rank;

2. S does not generically evince ranks;

3. $S$ does not totally evince ranks;

4. $Y^{\prime} \cong \mathbb{P}^{1}$.

Proof. Since any two distinct points of $\mathbb{P}^{N}$ are linearly independent (i.e., $\langle S\rangle$ is a line) and $v(Y)$ is the set of all points with $v(Y)$-rank $1, S$ evinces no rank if and only if $\langle S\rangle \subset v(Y)$. Use the fact that the lines of $v(Y)$ are contained in one of the factors of $v(Y)$. Since $v(Y)$ is cut out by quadrics, if $\langle S\rangle \nsubseteq v(Y)$, then $|\langle S\rangle \cap v(Y)| \leq 2$. Since $S \subset\langle S\rangle \cap v(Y)$, we see that all points of $\langle S\rangle \backslash S$ have rank 2

Proposition 4. Take $S \subset v(Y)$ with $|S|=3$ and $v(S)$ linearly independent. Write $S=v(A)$ with $A \subset Y^{\prime}$. Let $Y^{\prime}$ be the minimal multiprojective subspace of $Y$ containing $A$. Write $Y^{\prime}=\mathbb{P}^{m_{1}} \times \cdots \mathbb{P}^{m_{s}}$ with $s \geq 1$ and $m_{1} \geq \cdots \geq m_{s}>0$. We have $m_{1} \leq 2$.

If $\eta_{i \mid A}$ is injective for all $i$ and either $m_{2}=2$ or $s \geq 4$ or $m_{1}=2$ and $s=3$, then $S$ totally evinces its ranks. In all other cases for a general $E \in Y^{\prime}$ with $|E|=3, v(E)$ does not generically evince its ranks.

Proof. If $\eta_{i \mid A}$ is not injective for some $i$, then $S$ evinces no rank by Lemma 4 . Thus, we may assume that each $\eta_{i \mid A}$ is injective for all $i$. Each factor of $Y^{\prime}$ is the linear span of $\pi_{i}(A)$ in $\mathbb{P}^{n_{i}}$. Hence, $m_{1} \leq 2$. 
Omitting all factors which are points, we get the form of $Y^{\prime}$ we use. If $Y^{\prime}=\mathbb{P}^{1}$ (resp. $\mathbb{P}^{2}$, resp. $\mathbb{P}^{1} \times \mathbb{P}^{1}$ ), then each point of $\langle S\rangle$ has rank 1 (resp. 1, resp. $\leq 2$ ). Thus, in these cases, $S$ evinces no rank. If either $Y^{\prime}=\mathbb{P}^{2} \times \mathbb{P}^{1}$ or $Y^{\prime}=\left(\mathbb{P}^{1}\right)^{3}$, then $\sigma_{2}\left(\mathbb{P}^{2} \times \mathbb{P}^{1}\right)=\mathbb{P}^{5}$ and $\sigma_{2}\left(\left(\mathbb{P}^{1}\right)^{3}\right)=\mathbb{P}^{7}([23,26])$. Thus, the last assertion of the proposition is completed.

(a) Assume $s \geq 2$ and $m_{2}=2$. Taking a projection onto the first two factors, we reduce to the case $s=2$ (this reduction step is used only to simplify the notation). Take a $H \in\left|\mathcal{O}_{Y^{\prime}}(1,0)\right|$ containing $B$ (this is possible because $\left.h^{0}\left(\mathcal{O}_{\mathbb{P}^{2}}(1)\right)=3>|B|\right)$. Since $Y^{\prime}$ is the minimal multiprojective subspace of $Y$ containing $A$, we have $A \backslash A \cap H \neq \varnothing$. Since $B \backslash B \cap H=\varnothing$, (Ref. [52] Lemma 5.1) gives $h^{1}\left(\mathcal{I}_{A \backslash A \cap H}(0,1)\right)>0$. Thus, either there is $A^{\prime} \subset A$ with $\left|A^{\prime}\right|=2$ and $\eta_{1 \mid A^{\prime}}$ not injective (we excluded this possibility) or $|A \backslash A \cap H|=3$ (i.e., $A \cap H=\varnothing$ ) and $\eta_{1}(A) \subset \mathbb{P}^{2}$ is contained in a line $R$. Set $M:=\mathbb{P}^{2} \times R$. We get $A \subset M$ and hence $A$ is a contained in a proper multiprojective subspace, contradicting the definition of $Y^{\prime}$.

(b) Assume $s \geq 3$ and $m_{1}=2$. By part (a), we may assume $m_{2}=1$. Taking a projection, we reduce to the case $s=3$, i.e., $Y^{\prime}=\mathbb{P}^{2} \times \mathbb{P}^{1} \times \mathbb{P}^{1}$. Take $H$ as in step (a). As in step (a), we get $A \cap H=\varnothing$ and $\eta_{1}(A)$ contained in a line $R$ of the Segre embedding of $\mathbb{P}^{1} \times \mathbb{P}^{1}$, contradicting the definition of $Y^{\prime}$.

(c) Assume $s \geq 4$. By step (b), we may assume $m_{1}=1$. Taking a projection onto the first four factors of $Y^{\prime}$, we reduce to the case $Y^{\prime}=\left(\mathbb{P}^{1}\right)^{4}$. Fix any $H \in\left|\mathcal{O}_{Y^{\prime}}(1,1,0,0)\right|$ containing $B$. Assume for the moment $A \nsubseteq H$. By ([52] Lemma 5.1), we have $h^{1}\left(\mathcal{I}_{A \backslash A \cap H}(0,0,1,1)\right)>0$, i.e., either there are $a=\left(a_{1}, a_{2}, a_{3}, a_{4}\right) \in A, b=\left(b_{1}, b_{2}, b_{3}, b_{4}\right) \in A$ with $a \neq b$ and $\left(a_{3}, a_{4}\right)=\left(b_{3}, b_{4}\right)$ of $A \cap H=\varnothing$ and the projection of $A$ onto the last 2 factors of $Y^{\prime}$ is contained in a line. The last possibility is excluded by the minimality of $Y^{\prime}$. Thus, $a, b \in A$ exists. Set $A:=\{a, b, c\}$ and write $c=\left(c_{1}, c_{2}, c_{3}, c_{4}\right)$. Permuting the factors of $Y^{\prime}$, we see that, for each $E \subset\{1,2,3,4\}$, there is $A_{E} \subset A$ with $\left|A_{E}\right|=2$ and $\pi_{E}\left(A_{E}\right)$ is a singleton, where $\pi_{E}: Y^{\prime} \rightarrow \mathbb{P}^{1} \times \mathbb{P}^{1}$ denote the projection onto the factors of $Y^{\prime}$ corresponding to $E$. Since the cardinality of the set $\mathcal{S}$ of all subset of $\{1,2,3,4\}$ with cardinality 2 is larger than the cardinality of the set of all subsets of $A$ with cardinality 3 , there are $E, F \in \mathcal{S}$ such that $E \neq F$ and $A_{E}=A_{F}$. If $E \cap F \neq \varnothing$, say $E \cap F=\{i\}$, then $\eta_{i \mid A}$ is not injective, contradicting our assumption. If $E \cap F=\varnothing$, we have $E \cup F=\{1,2,3,4\}$. Since $A_{E}=A_{F}$, we get $\left|A_{E}\right|=1$, a contradiction.

Remark 4. Take a finite $S \subset v(Y)$ and fix $q \in\langle v(S)\rangle^{\prime}$. Let $A \subset Y$ be the subset with $v(A)=S$. It is easier to prove that $S$ evinces the rank of $q$ if we know that the minimal multiprojective subspace of $Y$ containing $A$ is the minimal multiprojective subspace $Y^{\prime \prime}$ of $Y$ with $q \in\left\langle v\left(Y^{\prime \prime}\right)\right\rangle$. Note that this is always true if $Y^{\prime \prime}=Y$, i.e., if the tensor $q$ is concise.

\section{Questions on the Case of Veronese Varieties}

Let $r_{\max }(n, d)$ denote the maximum of all $X_{n, d}$-ranks (in $[55,56]$ it is denoted with $r_{\max }(n+$ $1, d)$ ). The integer $r_{\max }(n, d)$ depends on two variables, $n$ and $d$. In this section, we ask some question on the asymptotic behavior of $r_{\max }(n, d)$ when we fix one variable, while the other one goes to $+\infty$.

Let $r_{\text {gen }}(n, d)$ denote the $X_{n, d}$-rank of a general $q \in \mathbb{P}^{r}$. These integers do not depend on the choice of the algebraically closed base field $\mathbb{K}$ with characteristic 0 . The diagonalization of quadratic forms gives $r_{\max }(n, 2)=r_{\text {gen }}(n, 2)=n+1$. The integers $r_{\text {gen }}(n, d), d>2$, are known by an important theorem of Alexander and Hirschowitz ([8-13]); with four exceptional cases, we have $r_{\text {gen }}(n, d)=\left\lceil\left(\begin{array}{c}n+d \\ n\end{array}\right) /(n+1)\right\rceil$. An important theorem of Blekherman and Teitler gives $r_{\max }(n, d) \leq 2 r_{\text {gen }}(n, d)$ (and even $r_{\max }(n, d) \leq 2 r_{\text {gen }}(n, d)-1$ with a few obvious exceptions) $([57,58])$. In particular, for a fixed $n$, we have

$$
\frac{1}{(n+1) !} \leq \liminf _{d \rightarrow+\infty} r_{\max }(n, d) / d^{n} \leq \limsup _{d \rightarrow+\infty} r_{\max }(n, d) / d^{n} \leq \frac{2}{(n+1) !} .
$$


It is reasonable to ask if $\liminf _{d \rightarrow+\infty} r_{\max }(n, d) / d^{n}$ exists and its value. Of course, it is tempting also to ask a more precise information about $r_{\max }(n, d)$ for $d \gg 0$. In the case $n=2$, De Paris proved in $[55,56]$ that $r_{\max }(2, d) \geq\left\lfloor\left(d^{2}+2 d+5\right) / 4\right\rfloor([56]$ Theorem 3$)$, which equality holds if $d$ is even ([56] (Proposition 2.4)) and suggested that equality holds for all $d$. Since $r_{\max }(2, d+1) \geq$ $r_{\max }(2, d)$ even for odd $d$, the integer $r_{\max }(2, d)$ grows like $d^{2} / 4$. Thus, there is an interesting interval between the general upper bound of [57] (which, in this case, has order $d^{2} / 3$ ) and $r_{\max }(2, d)$. There are very interesting upper bounds for the dimensions of the set of all points with rank bigger than the generic one ([59]).

What are

$$
\limsup _{n \rightarrow+\infty} \frac{(n+1) ! r_{\max }(n, d)}{d^{n}} \text { and } \liminf _{n \rightarrow+\infty} \frac{(n+1) ! r_{\max }(n, d)}{d^{n}} ?
$$

For all $d \geq 3$, study $r_{\max }(n, d)-r_{\max }(n, d-1)$ and compare for $d \gg 0 r_{\max }(n, d)-r_{\max }(n, d-1)$ with $r_{\max }(n-1, d)$ and $r_{\text {gen }}(n-1, d)$. Of course, this is almost exactly known when $n=2$ by Sylvester's theorem ([48]) and De Paris $([55,56])$, but $r_{\max }(2, d)-r_{\max }(2, d-1)$ for $d \gg 0$ is both $\sim r_{\text {gen }}(1, d)$ and $\sim r_{\max }(1, d) / 2$ and so we do not have any suggestion for the case $n>2$.

Funding: The author was partially supported by MIUR and GNSAGA of INdAM (Italy).

Conflicts of Interest: The author declares no conflict of interest.

\section{References}

1. Landsberg, J.M. Tensors: Geometry and Applications Graduate Studies in Mathematics; American Mathematical Society: Kingston, ON, Canada; New York, NY, USA, 2012; Volume 128.

2. Iarrobino, A.; Kanev, V. Lecture Notes in Mathematics. In Power Sums, Gorenstein Algebras, and Determinantal Loci; Springer-Verlag: Berlin, Germany; New York, NY, USA, 1999; Volume 1721.

3. Comon, P. Tensor decompositions: state of the art and applications, Mathematics. In Signal Processing, $V$ (Coventry, 2000); McWhirter, J.G., Proudler, I.K., Eds.; Clarendon Press: Oxford, UK, 2002; Volume 71, pp. 1-24.

4. Kolda, T.G.; Bader, B.W. Tensor decomposition and applications. SIAM Rev. 2009, 51, 455-500. [CrossRef]

5. Lickteig, T. Typical tensorial rank. Linear Algebra Appl. 1985, 69, 95-120. [CrossRef]

6. Lim, L.H.; Comon, P. Multiarray signal processing: Tensor decomposition meets compressed sensing. C. R. Mecanique 2010, 338, 311-320. [CrossRef]

7. Lim, L.H.; de Silva, V. Tensor rank and the ill-posedness of the best low-rank approximation problem. SIAM J. Matrix Anal. Appl. 2008, 30, 1084-1127.

8. Alexander, J. Singularités imposables en position générale aux hypersurfaces de $\mathbb{P}^{n}$. Composi. Math. 1988, $68,305-354$.

9. Alexander, J.; Hirschowitz, A. Un lemme d'Horace différentiel: application aux singularité hyperquartiques de $\mathbb{P}^{5}$. J. Algebr. Geom. 1992, 1, 411-426.

10. Alexander, J.; Hirschowitz, A. La méthode d'Horace éclaté: application à l'interpolation en degré quatre. Invent. Math. 1992, 107, 585-602. [CrossRef]

11. Alexander, J.; Hirschowitz, A. Polynomial interpolation in several variables. J. Algebr. Geom. 1995, 4, 201-222.

12. Brambilla, M.C.; Ottaviani, G. On the Alexander-Hirschowitz Theorem. J. Pure Appl. Algebra 2008, 212, 1229-1251. [CrossRef]

13. Chandler, K. A brief proof of a maximal rank theorem for generic double points in projective space. Trans. Am. Math. Soc. 2000, 353, 1907-1920. [CrossRef]

14. Postinghel, E. A new proof of the Alexander-Hirschowitz interpolation theorem. Ann. Mat. Pura Appl. 2012, 191, 77-94. [CrossRef]

15. Abo, H.; Ottaviani, G.; Peterson, C. Induction for secant varieties of Segre varieties. Trans. Am. Math. Soc. 2009, 361, 67-792. [CrossRef] 
16. Aladpoosh, T.; Haghighi, H. On the dimension of higher secant varieties of Segre varieties $\mathbb{P}^{n} \times \cdots \times \mathbb{P}^{n}$. J. Pure Appl. Algebra 2011, 215, 1040-1052. [CrossRef]

17. Catalisano, M.V.; Geramita, A.V.; Gimigliano, A. Secant varieties of $\mathbb{P}^{1} \times \cdots \times \mathbb{P}^{1}$ (n-times) are NOT defective for $n \geq 5$. J. Algebr. Geom. 2011, 20, 295-327. [CrossRef]

18. Abo, H. On non-defectivity of certain Segre-Veronese varieties. J. Symb. Comput. 2010, 45, 1254-1269. [CrossRef]

19. Abo, H.; Brambilla, M.C. Secant varieties of Segre-Veronese varieties $\mathbb{P}^{m} \times \mathbb{P}^{n}$ embedded by $\mathcal{O}(1,2)$. Exp. Math. 2009, 18, 369-384. [CrossRef]

20. Ab, H.; Brambilla, M.C. New examples of defective secant varieties of Segre-Veronese varieties. Collect. Math. 2012, 63, 287-297. [CrossRef]

21. Abo, H.; Brambilla, M.C. On the dimensions of secant varieties of Segre-Veronese varieties. Ann. Mat. Pura Appl. 2013, 192, 61-92. [CrossRef]

22. Baur, K.; Draisma, J.; de Graaf, W. Secant dimensions of minimal orbits: computations and conjectures. Exp. Math. 2007, 16, 239-250. [CrossRef]

23. Baur, K.; Draisma, J. Secant dimensions of low-dimensional homogeneous varieties. Adv. Geom. 2010, 10, 1-29. [CrossRef]

24. Catalisano, M.V.; Geramita, A.V.; Gimigliano, A. On the rank of tensors, via secant varieties and fat points. In Zero-Dimensional Schemes and Applications (Naples, 2000); Landsberg, J.M., Ed.; American Mathematical Society: Kingston, ON, Canada; New York, NY, USA, 2002; Volume 123, pp. 133-147.

25. Catalisano, M.V.; Geramita, A.V.; Gimigliano, A. Ranks of tensors, secant varieties of Segre varieties and fat points. Linear Algebra Appl. 2002, 355, 263-285. [CrossRef]

26. Catalisano, M.V.; Geramita, A.V.; Gimigliano, A. Segre-Veronese embeddings of $\mathbb{P}^{1} \times \mathbb{P}^{1} \times \mathbb{P}^{1}$ and their secant varieties. Collect. Math. 2007, 58, 1-24.

27. Laface, A.; Postinghel, E. Secant varieties of Segre-Veronese embeddings of $\left(\mathbb{P}^{1}\right)^{r}$. Math. Ann. 2013, 356, 1455-1470. [CrossRef]

28. Kruskal, J.B. Three-way arrays: rank and uniqueness of trilinear decompositions, with application to arithmetic complexity and statistics. Linear Algebra Appl. 1977, 18, 95-138. [CrossRef]

29. Bocci, C.; Chiantini, L.; Ottaviani, G. Refined methods for the identifiability of tensors. Ann. Mat. Pura Appl. 2014, 193, 1691-1702. [CrossRef]

30. Chiantini, L.; Ottaviani, G. On generic identifiability of 3-tensors of small rank. SIAM J. Matrix Anal. Appl. 2012, 33, 1018-1037. [CrossRef]

31. Chiantini, L.; Ottaviani, G.; Vanniuwenhoven, N. An algorithm for generic and low-rank specific identifiability of complex tensors. SIAM J. Matrix Anal. Appl. 2014, 35, 1265-1287. [CrossRef]

32. Chiantini, L.; Ottaviani, G.; Vanniuwenhoven, N. On identifiability of symmetric tensors of subgeneric rank. Trans. Amer. Math. Soc. 2017, 369, 4021-4042. [CrossRef]

33. Chiantini, L.; Ottaviani, G.; Vanniuwenhoven, N. Effective criteria for specific identifiability of tensors and forms. SIAM J. Matrix Anal. Appl. 2017, 38, 656-681. [CrossRef]

34. Domanov, I.; De Lathauwer, L. On the uniqueness of the canonical polyadic decomposition of third-order tensors-part I: Basic results and unique- ness of one factor matrix. SIAM J. Matrix Anal. Appl. 2013, 34, 855-875. [CrossRef]

35. Domanov, I.; De Lathauwer, L. On the uniqueness of the canonical polyadic decomposition of third-order tensors-Part II: Uniqueness of the overall decomposition. SIAM J. Matrix Anal. Appl. 2013, 34, 876-903. [CrossRef]

36. Domanov, I.; De Lathauwer, L. Generic uniqueness conditions for the canonical polyadic decomposition and INDSCAL. SIAM J. Matrix Anal. Appl. 2015, 36, 1567-1589. [CrossRef]

37. Massarenti, A.; Mella, M.; Staglian'o, G. Effective identifiability criteria for tensors and polynomials. J. Symbolic Comput. 2018, 87, 227-237. [CrossRef]

38. Sidiropoulos, N.D.; Bro, R. On the uniqueness of multilinear decomposition of N-way arrays. J. Chemom. 2000, 14, 229-239. [CrossRef]

39. Abo, H.; Ottaviani, G.; Peterson, C. Non-defectivity of Grassmannians of planes. J. Algebr. Geom. 2012, 21, 1-20. [CrossRef] 
40. Araujo, C.; Massarenti, A.; Rischter, R. On nonsecant defectivity of Segre-Veronese varieties. arXiv 2016, arXiv:1611.01674

41. Boralevi, A. A note on secants of Grassmannians. Rend. Istit. Mat. Univ. Trieste 2013, 45, 67-72.

42. Buczyńska, W.; Buczyński, J. Secant varieties to high degree Veronese reembeddings, catalecticant matrices and smoothable Gorenstein schemes. J. Algebr. Geom. 2014, 23, 63-90. [CrossRef]

43. Buczyński, J.; Ginensky, A.; Landsberg, J.M. Determinantal equations for secant varieties and the Eisenbud-Koh-Stillman conjecture. J. Lond. Math. Soc. 2013, 88, 1-24. [CrossRef]

44. Buczyński, J.; Landsberg, J.M. Ranks of tensors and a generalization of secant varieties. Linear Algebra Appl. 2013, 438, 668-689. [CrossRef]

45. Chiantini, L.; Ciliberto, C. On the dimension of secant varieties. J. Europ. Math. Soc. 2006, 73, $436-454$. [CrossRef]

46. Draisma, J. A tropical approach to secant dimension. J. Pure Appl. Algebra 2008, 212, 349-363. [CrossRef]

47. Landsberg, J.M.; Teitler, Z. On the ranks and border ranks of symmetric tensors. Found. Comput. Math. 2010, 10, 339-366. [CrossRef]

48. Comas, G.; Seiguer, M. On the rank of a binary form. Found. Comp. Math. 2011, 11, 65-78. [CrossRef]

49. Piene, R. Cuspidal projections of space curves. Math. Ann. 1981, 256, 95-119. [CrossRef]

50. Ballico, E.; Bernardi, A. Decomposition of homogeneous polynomials with low rank. Math. Z. 2012, 271, 1141-1149. [CrossRef]

51. Bernardi, A.; Gimigliano, A.; Idà, M. Computing symmetric rank for symmetric tensors. J. Symb. Comput. 2011, 46, 34-53. [CrossRef]

52. Ballico, E.; Bernardi, A. Stratification of the fourth secant variety of Veronese variety via the symmetric rank. Adv. Pure Appl. Math. 2013, 4, 215-250. [CrossRef]

53. Ballico, E. Finite subsets of projective spaces with bad postulation in a fixed degree. Beitrage zur Algebra und Geometrie 2013, 54, 81-103 [CrossRef]

54. Ballico, E. Finite defective subsets of projective spaces. Riv. Mat. Univ. Parma 2013, 4, 113-122.

55. De Paris, A. The asymptotic leading term for maximum rank for ternary forms of a given degree. Linear Algebra Appl. 2016, 500, 15-29. [CrossRef]

56. De Paris, A. High-rank ternary forms of even degree. Arch. Math. 2017, 109, 505-510. [CrossRef]

57. Blekherman, G.; Teitler, Z. On maximum, typical and generic ranks. Math. Ann. 2015, 362, 1021-1031. [CrossRef]

58. Blekherman, G.; Teitler, Z. Some examples of forms of high rank. Collect. Math. 2016, 67, 431-441.

59. Buczyński, J.; Han, K.; Mella, M.; Teitler, Z. On the locus of points of high rank. Eur. J. Math. 2018, 4, 113-136. [CrossRef]

(C) 2018 by the author. Licensee MDPI, Basel, Switzerland. This article is an open access article distributed under the terms and conditions of the Creative Commons Attribution (CC BY) license (http:/ / creativecommons.org/licenses/by/4.0/). 